\title{
La flore du conservatoire botanique Michel Adanson de Mbour (Sénégal) : perspective pour un plan d'aménagement et de gestion
}

\author{
Diop R.D., Mbaye M.S., Noba K.
}

Laboratoire de Botanique et de Biodiversité, Département de Biologie Végétale, Faculté des Sciences et Techniques, Université Cheikh Anta DIOP, B.P.5005 Dakar-Fann, Sénégal.

Auteur correspondant/ Corresponding author : Richard Demba DIOP

Tel : +221774378034 ,Email : richarddiop2004@yahoo.fr

Original submitted in on $13^{\text {th }}$ January 2017. Published online at www.m.elewa.orgon $31^{\text {st }}$ January 2017 http://dx.doi.org/10.4314/jab.v109i1.13

\section{RESUME}

Objectif : Cette étude a été réalisée dans la commune de Mbour dans le but de caractériser la flore du conservatoire botanique Michel ADANSON. Elle se propose d'établir la composition spécifique, les spectres taxonomique, biologique et chorologique et d'apprécier la fréquence et l'abondance des espèces.

Méthodologie et résultats : Des relevés phytosociologiques ont été réalisées en 2010 dans le conservatoire. Au total 140 espèces ont été recensées, réparties dans 115 genres et 53 familles. Les Dicotylédones représentent $90,57 \%$ des espèces de la flore contre $9,43 \%$ de Monocotylédones. La plupart des espèces sont des nanophanerophytes $(47,86 \%)$ et des thérophytes $(47,86 \%)$. Cette flore est aussi marquée par la prédominance des espèces africaines $(33,8 \%)$ et des espèces pantropicales $(21,6 \%)$.Plus de la moitié des espèces $(59 \%)$ recensées sont rares ou accidentelles du fait du caractère du site qui est un conservatoire. Environ $7,86 \%$ des espèces du conservatoire sont qualifiées d'espèces constantes. Nous avons $2,26 \%$ des espèces qui ont un recouvrement moyen de $32 \%$, ces espèces sont les plus abondantes du conservatoire.

Conclusion et application : Ce travail a permis de déterminer la structure du conservatoire botanique Michel ADANSON de Mbour. En effet, les spectres taxonomique, botanique, et chorologique sont déterminés de même que la fréquence des espèces et leur abondance dominance. Ces résultats sont importants pour la mise en place d'un plan d'aménagement et de gestion du conservatoire.

Mots clés : flore, végétation, Conservatoire botanique, ENDA, Mbour

\section{ABSTRACT}

The flora of the Michel Adanson botanical conservatory Mbour (Senegal): perspective for management and development.

Objective : This study was conducted in the town of Mbour in order to characterize the flora of the botanical conservatory Michel Adanson. It proposes to establish the species composition, the taxonomic spectra, biological and chorological and appreciate the frequency and abundance of species.

Methodology and Results: Phytosociological surveys were conducted in 2010 in the conservatory. In total 140 species were recorded, distributed in 115 genera and 53 families. Dicotyledons represent $90.57 \%$ of the species of flora against $9.43 \%$ of Monocotyledons. Most species are nanophanerophytes $(47.86 \%)$ and 
therophytes (47.86\%). This flora is also marked by the predominance of African species $(33.8 \%)$ and pantropical species $(21.6 \%)$. More than half of the species $(59 \%)$ surveyed are rare or accidental because of the character of the site which is a conservatory. Approximately $7.86 \%$ of the conservatory species are referred to constant species. There are $2.26 \%$ of the species that have an average recovery of $32 \%$, those species are the most abundant of the conservatory.

Conclusion and Application: This work has determined the structure of the botanical conservatory Michel Adanson Mbour. Indeed, the taxonomic spectra, botany, and chorological are determined as well as the frequency and abundance of species dominance. These results are important for the establishment of a management plan and interim management.

Keywords : flora, vegetation, Botanical Conservatory, ENDA, Mbour

\section{INTRODUCTION}

Les dernières décennies ont été marquées par une insuffisance chronique des pluies dans le sahel. Ce déficit hydrique auquel, s'ajoutent une mauvaise répartition des pluies et de nombreux autres facteurs, contribue à la désertification des marges du Sahara (Kahlem, 1981).L'impact de ces contraintes s'est manifesté d'une part par une réduction considérable des superficies boisées, et d'autre part par une modification de la structure des formations végétales et une raréfaction voire une disparition de certaines espèces (Sambou, 2004). A certains endroits, ces facteurs ont presque compromis les possibilités de reconstitution de la végétation. Or, la végétation naturelle est une importante source pour l'alimentation, la santé et la satisfaction des besoins énergétiques des populations dans les pays du Sahel (Sambou, 2004). Au Sénégal, cette dégradation n'a pas épargné les formations végétales des aires protégées. Conscientes de cette problématique, les organisations soucieuses de la conservation des ressources naturelles et de la biodiversité comme ENDA ont mis en place des programmes pour promouvoir une utilisation durable

\section{MATERIEL ET METHODES}

Présentation de la zone d'étude : Le conservatoire botanique Michel Adanson (Fig.1) $\left(14^{\circ} 25^{\prime} 14.40^{\prime \prime} \mathrm{N}\right.$ et $16^{\circ} 59^{\prime} 20.19^{\prime \prime} 0$ ) est localisé à Mbour. II s'étend sur 4 hectares autour du Centre d'Application au Développement Intégré (CADI) qui a une superficie de 4,97 ha. II est situé à $82 \mathrm{~km}$ au sud de Dakar, dans un quartier de la ville de Mbour, dénommé Grand Mbour, en bordure de mer (Enda-Madesahel, 2003). La commune de Mbour (Fig.2) couvre une superficie de $1725 \mathrm{~km}^{2}$. Chef-lieu du département qui porte le même nom, elle est localisée entre le Cap-Vert et la pointe de Sangomar en des ressources végétales et surtout des plantes médicinales pour subvenir aux problèmes de santé des populations. La création du Conservatoire botanique Michel Adanson dans le Centre d'Application au Développement Intégré (CADI) Seydou Nourou TALL à Mbour répond à ce souci de développement durable des communautés de base. Face à l'ampleur de ce processus de disparation des plantes médicinales, Enda-madesahel (Méthodes appliquée au Développements de Sahel) a pris l'initiative de mettre sur pied un conservatoire botanique des plantes médicinales. Cependant, l'insuffisance voire l'absence de données scientifiques sur la flore du CADI notamment au niveau du Conservatoire Michel Adanson est une contrainte forte qui limite la mise en œuvre des plans d'aménagement et de gestion indispensables à I'utilisation rationnelle des ressources du Conservatoire. C'est dans ce contexte qu'il faut situer cette étude qui viseà contribuer à une meilleure connaissance des ressources naturelles végétales du conservatoire en vue de leur utilisation durable.

demi-cuvette fermée à l'Ouest par l'océan Atlantique. Cette collectivité est limitée au nord, à l'Est et au Sud par la communauté rurale de Malicounda. Sa localisation en bordure de mer entre le Cap-Vert et la Pointe de Sangomar lui confère de nombreux atouts (pèche et tourisme) qui expliquent son poids démographique (Tine, 2009). Les sols sont majoritairement de type Dior dans le département (plus de 50\%), avec une partie latéritique dans la communauté rurale de Diass et à Sindia. Une partie Deck-dior à caractéristique plus prononcée de Deck est également notable à Mbodjène (communauté 
rurale de Ngéniène). Le cumul annuel des pluies des années 2008 et 2009 fut respectivement $605,4 \mathrm{~mm}$, étalé sur 60 jours et, $673,2 \mathrm{~mm}$ pour 37 jours. Pour l'année 2010, ce cumul fut $907,6 \mathrm{~mm}$, pour 56 jours de pluies. Faute d'habitats, la faune sauvage est très peu représentée dans le département, même s'il faut reconnaître les efforts de réintroduction en grande et petite faune, dans la réserve de Bandia qui est un véritable exemple de conservation de la biodiversité faunique et floristique dans le département (Tine, 2009).

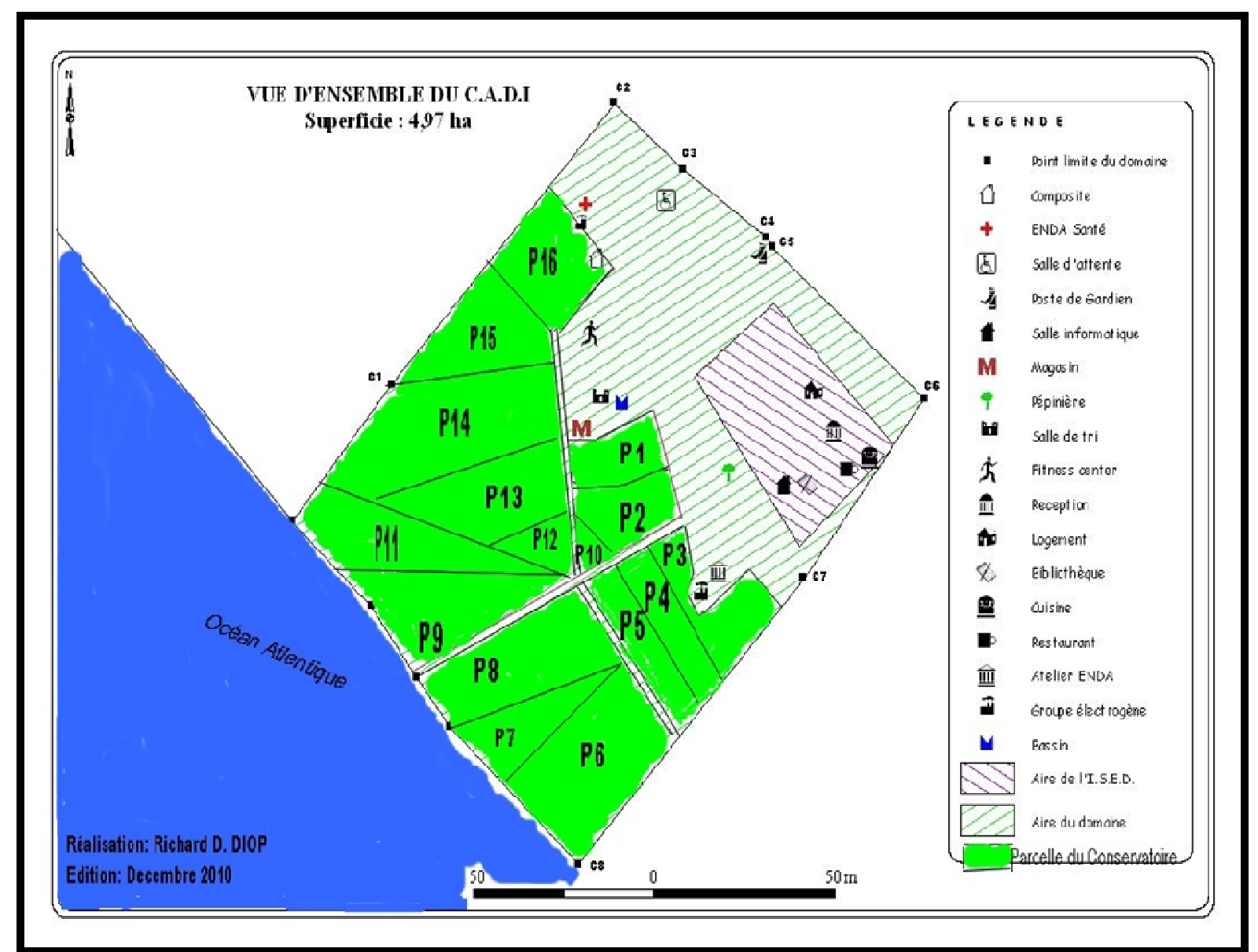

Figure 1 : Carte du centre d'application au développement intégré (C.A.D.I.) 


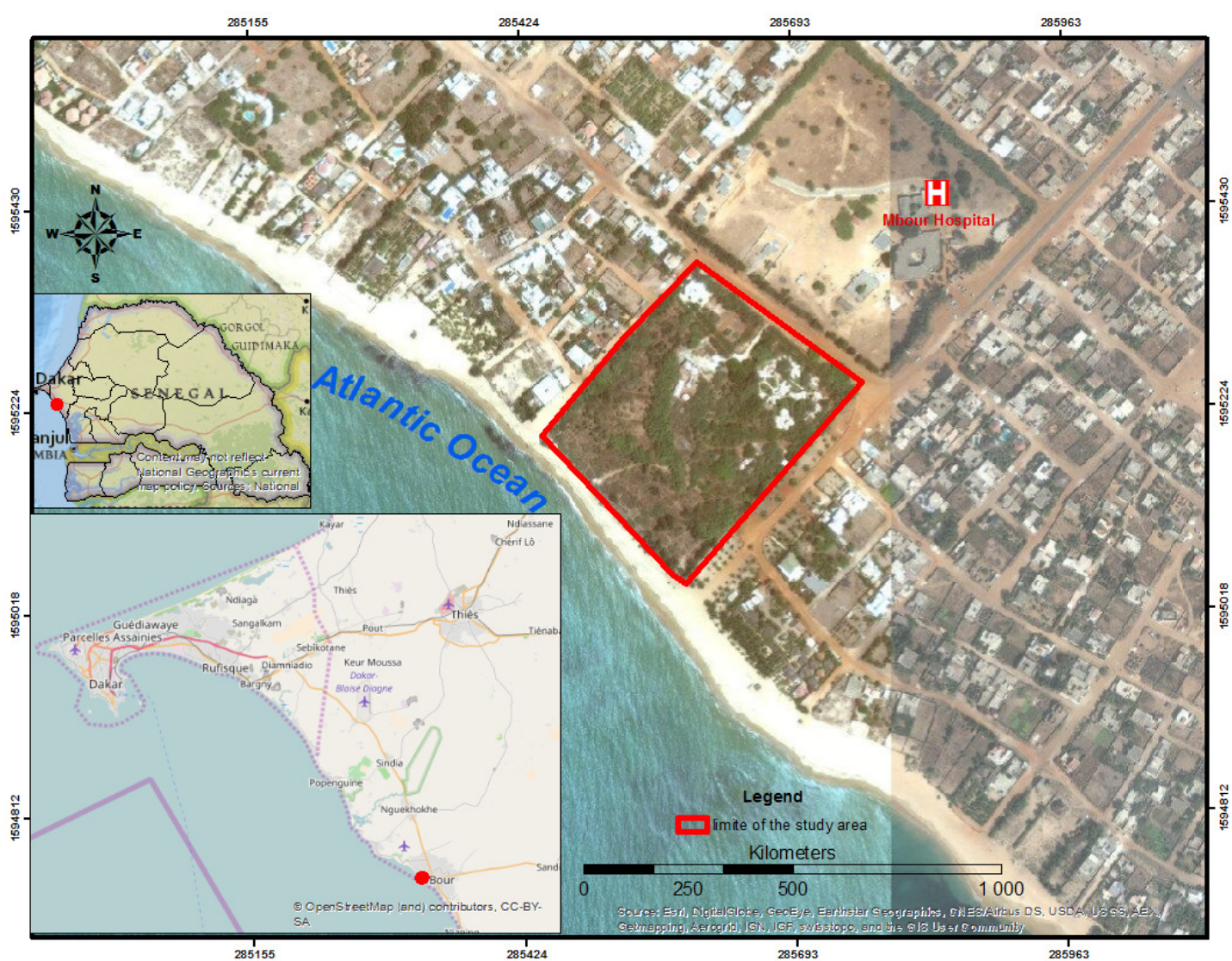

Figure 2 : Situation géographique de la commune de Mbour et du C.A.D.I. (source : Google earth, 2016)

Étude la flore: Cette étude a été réalisée dans le Conservatoire botanique Michel Adanson de Mbour en 2010. Elle a consisté en l'inventaire des espèces végétales des 16 parcelles du conservatoire. Pour cette étude, des relevés phytosociologiques ont été réalisés en appliquant la technique du «tour de champs». Cette méthode de prospection itinérante consiste à inventorier toutes les espèces en parcourant une surface d'observation, définie en fonction de l'hétérogénéité des milieux, dans différentes directions (Noba, 2002). La détermination des espèces a été effectuée à l'aide des flores du Sénégal (Berhaut 1967,1971-1991; Flora of West tropical Africa de Hutchison \& Dazeil 1954, 1963,1968; Mugnier 2008), d'échantillons de l'herbier du Département de Biologie Végétale de la Faculté des Sciences et Techniques de l'Université Cheikh Anta DIOP de Dakar. Les nomenclatures employées sont celles de Lebrun \& Stork $(1991,1992,1995,1997)$ et la nouvelle classification APG III au niveau des familles. Pour les types biologiques, la classification de Raunkiaer (1934) plus adaptée à la zone tropicale ou à la saison sèche $a$ été utilisée. Cette classification distingue six (6) formes biologiques qui sont les nanophanerophytes $(P)$, les chaméphytes (C), les hemicryptophytes (Ch), les géophytes $(G)$, les thérophytes $(T)$ et les plantes parasites (Par.). Pour la répartition géographique, les informations proviennent essentiellement des flores: Hutchinson \&Dalziel $(1954,1963,1968)$. A partir des relevés, la fréquence ou distribution des espèces dans le peuplement et l'indice d'abondance moyen seront calculés. L'indice d'abondance dominance moyen (ADM) est calculé par rapport au nombre de relevés dans lesquels l'espèce est présente; pour le calcul de cet indice, les codes semi quantitatifs d'abondance/dominance ( $A D$ code) sont rendus quantitatifs (AD num) (Gillet, 2000). L'échelle de BraunBlanquet n'est pas linéaire, chaque code est converti en un recouvrement moyen, à l'aide d'une table de correspondance (tableau1) (Gillet, 2000). L'analyse quantitative de la structure de la végétation a été réalisée à l'aide de la méthode de Caratini (1985) selon le tableau 2. 
Diop et al., J. Appl. Biosci. 2017 La flore du conservatoire botanique Michel Adanson de Mbour (Sénégal) : perspective pour un plan d'aménagement et de gestion

Tableau 1: Table de correspondance des codes d'abondance dominance et de recouvrement

\begin{tabular}{|c|c|c|c|c|}
\hline AD code & AD num & Rec. Moy & Rec. Min & Rec. Max \\
\hline$R$ & 0,1 & 0,03 & 0 & 0,1 \\
\hline+ & 0,5 & 0,3 & 0,1 & 1 \\
\hline 1 & 1 & 3 & 1 & 5 \\
\hline 2 & 2 & 14 & 5 & 25 \\
\hline 3 & 3 & 32 & 25 & 50 \\
\hline 4 & 4 & 57 & 50 & 75 \\
\hline 5 & 5 & 90 & 75 & 100 \\
\hline
\end{tabular}

Tableau 2: Table de correspondance des indices de fréquences et leurs qualifications

\begin{tabular}{|c|c|c|}
\hline Fréquences & Indices & Qualifications \\
\hline 0,8 à 1 & V & Constante \\
\hline 0,6 à 0,8 & IV & Abondante \\
\hline 0,4 à 0,6 & III & Fréquente \\
\hline 0,2 à 0,4 & II & Accessoire \\
\hline 0 à 0,2 & I & Rare ou Accidentelle \\
\hline
\end{tabular}

\section{RÉSULTATS}

Composition spécifique : Le tableau 3 présente les différentes espèces et familles rencontrées dans le Conservatoire botanique Michel Adanson de Mbour avec des indications sur le type biologique et l'appartenance biogéographique de chaque espèce. La flore du conservatoire botanique Michel ADANSON est composée de 140 espèces réparties dans 115 genres et 53 familles (Tab. 4). Les espèces de cette flore appartiennent à un embranchement, celui des Spermaphytes et un sous embranchement celui des Angiospermes.

Spectre taxonomique: Le tableau 5 donne des indications sur la structure de la flore du Conservatoire. II apparaît ainsi qu'au plan de la diversité, la flore du
Conservatoire botanique Michel Adanson de Mbour est composée uniquement d'angiospermes. Les familles appartenant à la classe des dicotylédones représentent plus $80 \%$. Ces proportions restent quasiment les mêmes en ce qui concerne la diversité des genres. Pour ce qui est de la diversité au niveau spécifique, les dicotylédones représentent $85,71 \%$. Dans la flore du Conservatoire botanique Michel Adanson de Mbour (Tab. 3), sept familles regroupent plus de la moitié $(50,4 \%)$ des espèces recensées. Ce sont les Fabaceae (19,4\%), Poaceae $(9,4 \%)$, Malvaceae $(6,5 \%)$, Euphorbiaceae $(5,0 \%)$, Convolvulaceae (4,3\%), Amaranthaceae (2,9\%), Capparidaceae $(2,9 \%)$.

Tableau 3: Liste des espèces recensées avec des indications sur leur type biologique (T.B), leur répartition géographique (R.G)

\begin{tabular}{|l|l|l|l|}
\hline Familles & Espèces & T.B. & R.G. \\
\hline \multirow{3}{*}{ ACANTHACEAE (D) } & Justicia kotschyi (Hochst.) Dandy & T & Af \\
\cline { 2 - 4 } & Monechma ciliatum (Jacq.) Miln.-Red & T & Af \\
\cline { 2 - 4 } & Peristrophe paniculata (Forssk.) Brum. & T & As \\
\hline \multirow{5}{*}{ AMARANTHACEAE (D) } & Achyranthes argentea Lam. & T & Cosm \\
\cline { 2 - 4 } & Amaranthus hibridus L. & T & Cosm \\
\cline { 2 - 4 } & Amaranthus spinosus L. & T & Pt \\
\cline { 2 - 4 } & Pupalia lappacea (L.) Juss & T & Mas \\
\hline \multirow{2}{*}{ ANACARDIACEAE (D) } & Mangifera indica L. & $\mathrm{P}$ & $\mathrm{Pt}$ \\
\cline { 2 - 4 } & Sclerocarya birrea (A. Rich.) Hochst. & $\mathrm{P}$ & $\mathrm{Af}$ \\
\hline ANNONACEAE (D) & Annona squamosa L. & $\mathrm{P}$ & $\mathrm{Pt}$ \\
\hline \multirow{2}{*}{ ARECACEAE (M) } & Phoenix dactylifera L. & $\mathrm{P}$ & $\mathrm{As}$ \\
\cline { 2 - 4 } & Phoenix reclinata Jacq. & $\mathrm{P}$ & $\mathrm{Af}$ \\
\hline ASCLEPIADACEAE (D) & Calotropis procera (Ait.) Ait. f. & $\mathrm{P}$ & $\mathrm{As}$ \\
\hline
\end{tabular}


Diop et al., J. Appl. Biosci. 2017 La flore du conservatoire botanique Michel Adanson de Mbour (Sénégal) : perspective pour un plan d'aménagement et de gestion

\begin{tabular}{|c|c|c|c|c|}
\hline & Leptadenia hastata (Pers.) Decne. & $\mathrm{T}$ & Af \\
\hline & & Pergularia daemia (Forsk.) Chiov. & $\mathrm{T}$ & As \\
\hline \multirow{4}{*}{\multicolumn{2}{|c|}{ ASTERACEAE(D) }} & Artemisia annua L. & $\mathrm{P}$ & $\mathrm{Pt}$ \\
\hline & & Centaurea perrottetii DC. & $\mathrm{T}$ & Af \\
\hline & & Emilia sonchifolia (L.) DC. & $T$ & $\mathrm{Pt}$ \\
\hline & & Launaea intybacea (Jacq) Beauv & $\mathrm{T}$ & $\mathrm{Pt}$ \\
\hline \multicolumn{2}{|c|}{ BALANITACEAE (D) } & Balanites aegyptiaca (L.) Del. & $\mathrm{P}$ & As \\
\hline \multirow{2}{*}{\multicolumn{2}{|c|}{ BIGNONIACEAE (D) }} & Crescentia cujete L. & $\mathrm{P}$ & Am \\
\hline & & Kigelia africana (Lam.) Benth & $\mathrm{P}$ & Af \\
\hline & Heliotropium bacciferum Forsk. & $\mathrm{T}$ & Af \\
\hline \multicolumn{2}{|c|}{ BURSERARACEAE (D) } & Commiphora africana (A. Rich.) Engl. & $\mathrm{P}$ & Af \\
\hline \multicolumn{2}{|c|}{ CACTACEAE (D) } & Opuntia tuna (L.) Miller & $P$ & $\mathrm{Am}$ \\
\hline \multirow{4}{*}{\multicolumn{2}{|c|}{ CAPPARIDACEAE (D) }} & Boscia senegalensis (Pers.) Lam. & $\mathrm{Ch}$ & Af \\
\hline & & Cadaba farinosa Forsk. & $P$ & As \\
\hline & & Capparis tomenosa Lam. & $P$ & Af \\
\hline & & Crataeva religiosa Sieber & $P$ & Af \\
\hline \multicolumn{2}{|c|}{ CARICACEAE (D) } & Carica papaya $\mathrm{L}$. & $P$ & $\mathrm{Pt}$ \\
\hline \multicolumn{2}{|c|}{ CASUARINACEAE (D) } & Casuarina equisetifolia Forst. & $P$ & $\mathrm{Pt}$ \\
\hline \multicolumn{2}{|c|}{ CELASTRACEAE (D) } & Maytenus senegalensis (Lam.) Exell. & $P$ & Af \\
\hline \multirow{2}{*}{\multicolumn{2}{|c|}{ COMBRETACEAE (D) }} & Combretum aculeatum Vent. & $\mathrm{Ch}$ & Af \\
\hline & & Terminalia catappa L. & $P$ & $\mathrm{Pt}$ \\
\hline \multirow{2}{*}{\multicolumn{2}{|c|}{ COMMELINACEAE (M) }} & Commelina benghalensis L. & $\mathrm{T}$ & Af \\
\hline & & Commelina forskalaei Vahl. & $\mathrm{T}$ & Mas \\
\hline \multirow{6}{*}{\multicolumn{2}{|c|}{ CONVOLVULACEAE (D) }} & Ipomoea pes-tigridis L. & $T$ & As \\
\hline & & Ipomoea stolonifera (Cyrill.) J.F. Gmel & $\mathrm{T}$ & As \\
\hline & & Ipomoea coptica (L.) Roth ex Roem. et Schult. & $\mathrm{T}$ & Asu \\
\hline & & Jacquemontia tamnifolia (L.) Griseb. & $T$ & $\mathrm{Am}$ \\
\hline & & Merremia aegyptia (L.) Urban & $\mathrm{T}$ & Af \\
\hline & & Merremia tridentata (L.) Hall. $f$ & $\mathrm{~T}$ & Af \\
\hline \multirow{2}{*}{\multicolumn{2}{|c|}{ CUCURBITACEAE (D) }} & Coccinia grandis (L.) Voigt. & $\mathrm{T}$ & Af \\
\hline & & Momordica balsamina L. & $\mathrm{T}$ & As \\
\hline \multirow{2}{*}{\multicolumn{2}{|c|}{ CYPERACEAE (M) }} & Cyperus esculentus L. & G & Cosm \\
\hline & & Cyperus maritimus Poir. & G & $\mathrm{M}$ \\
\hline \multirow{7}{*}{\multicolumn{2}{|c|}{ EUPHORBIACEAE (D) }} & Croton lobatus L. & $\mathrm{T}$ & As \\
\hline & & Euphorbia balsamifera Ait. & $P$ & Ase \\
\hline & & Euphorbia hirta L. & $\mathrm{T}$ & $\mathrm{Pt}$ \\
\hline & & Euphorbia tirucalli L. & $\mathrm{P}$ & Mas \\
\hline & & Jatropha curcas L. & $P$ & Cosm \\
\hline & & Phyllenthus acidus (L.) Skeel. & $P$ & As \\
\hline & & Phyllenthus pentandrus Sch. \& Th. & $\mathrm{T}$ & Af \\
\hline \multirow{9}{*}{ 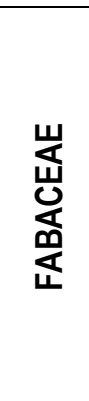 } & \multirow{9}{*}{ Caesalpinoideae(D) } & Bauhinia rufescens Lam. & $\mathrm{P}$ & Af \\
\hline & & Caesalpinia pulcherrima (L.) Sw & $P$ & As \\
\hline & & Cassia italica (Mill.) F.W. Andr. & $\mathrm{T}$ & As \\
\hline & & Cassia occidentalis L. & $\mathrm{T}$ & $\mathrm{Pt}$ \\
\hline & & Cassia siamea Lam. & $\mathrm{P}$ & $\mathrm{Pt}$ \\
\hline & & Griffonia simplicifolia (Vahl ex DC.) Baill. & $P$ & Af \\
\hline & & Parkinsonia aculeata L. & $P$ & Am \\
\hline & & Piliostigma reticulatum (DC.) Hochst. & $\mathrm{P}$ & Af \\
\hline & & Tamarindus indica L. & $\mathrm{P}$ & $\mathrm{Pt}$ \\
\hline
\end{tabular}


Diop et al., J. Appl. Biosci. 2017 La flore du conservatoire botanique Michel Adanson de Mbour (Sénégal) : perspective pour un plan d'aménagement et de gestion

\begin{tabular}{|c|c|c|c|c|}
\hline \multirow{6}{*}{\multicolumn{2}{|c|}{ Mimosoideae (D) }} & Acacia adansonii G. et Perr. & $\mathrm{P}$ & Af \\
\hline & & Acacia ataxacantha DC. & $\mathrm{P}$ & Af \\
\hline & & Acacia bivenosa DC. & $\mathrm{P}$ & Asu \\
\hline & & Acacia gourmaensis A Chev. & $\mathrm{P}$ & Af \\
\hline & & Acacia nilotica var nilotica (L.) Willd. ex Del. & $\mathrm{P}$ & Af \\
\hline & & Acacia tortilis subsp. raddiana (Savi) Brenan & $\mathrm{P}$ & As \\
\hline & & Faidherbia albida (Del.) A. Chev. & $\mathrm{P}$ & Af \\
\hline & & Leucaena leucocephala (Lam.) De Wit & $\mathrm{P}$ & Cosm \\
\hline & & Peltophorum pterocarpum (DC) K. Heyne & $\mathrm{P}$ & $\mathrm{Pt}$ \\
\hline & & Pithecellobium dulce (Roxb.) Benth. & $\mathrm{P}$ & Am \\
\hline & & Prosopis juliflora (Sw.) DC. & $\mathrm{P}$ & Cosm \\
\hline & \multirow{7}{*}{ Faboideae (D) } & Abrus precatorius L. & $\mathrm{Ch}$ & $\mathrm{Pt}$ \\
\hline & & Alysicarpus ovalifolius (S. \& Th.) Léon & $\mathrm{T}$ & $\mathrm{Pt}$ \\
\hline & & Canavalia rosea (Sw.) DC. & C & $\mathrm{Pt}$ \\
\hline & & Clitoria ternatea $\mathrm{L}$. & $T$ & Cosm \\
\hline & & Sesbania pachycarpa DC. & $T$ & Asu \\
\hline & & Tephrosia lathyroides G. \&Perr. & $T$ & $\mathrm{Am}$ \\
\hline & & Vigna gracilis (G. \&Perr.) Hook.f. & $T$ & Af \\
\hline \multicolumn{2}{|c|}{ HIPPOCRATEACEAE (D) } & Hippocratea myriantha Oliv. & $\mathrm{P}$ & Mas \\
\hline \multicolumn{2}{|c|}{ LABIEAE (D) } & Hyptis suaveolens Poit. & $T$ & Am As \\
\hline \multicolumn{2}{|c|}{ LILIACEAE(M) } & Scilla sudanica A. Chev. & G & Af \\
\hline \multicolumn{2}{|c|}{ LORANTHACEAE (D) } & Tapinaenthus bangwensis (Eng.et Kr.) Danzer & Par & Af \\
\hline \multicolumn{2}{|c|}{ LYTHRACEAE (D) } & Lawsonia inermis $\mathrm{L}$. & $P$ & Asu \\
\hline \multirow{9}{*}{ 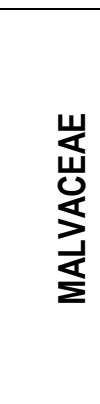 } & Bombacoideae (D) & Adansonia digitata L. & $\mathrm{P}$ & Af \\
\hline & \multirow{4}{*}{ Malvoideae (D) } & Gossypium barbadense L. & $\mathrm{P}$ & $\mathrm{Pt}$ \\
\hline & & Hibiscus mechowii Garcke & $T$ & Af \\
\hline & & Hibiscus physaloides G.\& Perr. & $T$ & Mas \\
\hline & & Hibiscus sabdariffa L. & $T$ & $\mathrm{Pt}$ \\
\hline & Sterculiaceae (D) & Guazuma ulmifolia Lam. & $\mathrm{P}$ & Am \\
\hline & \multirow{3}{*}{ Grewioideae (D) } & Corchorus tridens L. & $T$ & Asu \\
\hline & & Grewia bicolor Juss. & $\mathrm{P}$ & As \\
\hline & & Triumfetta pentandra A. Rich. & $T$ & Af \\
\hline \multicolumn{2}{|c|}{ MELIACEAE (D) } & Azadirachta indica A. Juss. & $\mathrm{P}$ & $\mathrm{Pt}$ \\
\hline \multicolumn{2}{|c|}{ MENISPERMACEAE (D) } & Tinospora bakis (A. Rich) Miers. & $\mathrm{P}$ & Af \\
\hline \multicolumn{2}{|c|}{ MORACEAE (D) } & Ficus vogelii (Miq.) Miq. & $\mathrm{P}$ & Af \\
\hline \multicolumn{2}{|c|}{ MORINGACEAE (D) } & Moringa oleifera Lam. & $\mathrm{P}$ & $\mathrm{Pt}$ \\
\hline \multirow{3}{*}{\multicolumn{2}{|c|}{ MYRTACEAE (D) }} & Eucalyptus alba Reinw. ex Blume & $\mathrm{P}$ & $\mathrm{Pt}$ \\
\hline & & Melaleuca leucadendron L. & $\mathrm{P}$ & Asu \\
\hline & & Syzygium guineense (Willd.) DC. & $\mathrm{P}$ & Af \\
\hline \multirow{3}{*}{\multicolumn{2}{|c|}{ NYCTAGINACEAE (D) }} & Boerhaavia diffusa $\mathrm{L}$. & $T$ & $\mathrm{Pt}$ \\
\hline & & Boerhaavia repens $\mathrm{L}$. & $T$ & $\mathrm{Pt}$ \\
\hline & & Bougainvillea spectabilis Willd & $\mathrm{P}$ & Am \\
\hline \multicolumn{2}{|c|}{ PASSIFLORACEAE (D) } & Passiflora foetida L. & $T$ & Am \\
\hline \multicolumn{2}{|c|}{ PEDALIACEAE (D) } & Ceratotheca sesamoides Endl. & $T$ & Af \\
\hline \multirow{5}{*}{\multicolumn{2}{|c|}{ POACEAE (M) }} & Brachiraria xantholeuca (Schinz) Stapf. & $T$ & Af \\
\hline & & Cenchrus biflorus Roxb & $T$ & As \\
\hline & & Chloris pilosa Sch. \&Thonn. & $\mathrm{T}$ & Af \\
\hline & & Cymbopogon giganteus Chiov. & $\mathrm{Ch}$ & Af \\
\hline & & Cynodon dactylon (L.) Pers. & C & Cosm \\
\hline
\end{tabular}




\begin{tabular}{|c|c|c|c|}
\hline & Dactyloctenium aegyptium (L.) Willd. & $\mid T$ & $\mathrm{Pt}$ \\
\hline & Digitaria exilis (Kippist) Stapf. & $\mathrm{T}$ & Af \\
\hline & Digitaria horizontalis Willd. & $\mathrm{T}$ & Am \\
\hline & Enteropogon prieurii (Kunth) Clayton & $T$ & As \\
\hline & Eragrostis ciliaris (L.) R. Br. & $\mathrm{T}$ & $\mathrm{Pt}$ \\
\hline & Pennisetum pedicellatumTrin. & $T$ & As \\
\hline & Pennisetum violaceum (Lam.) Rich & $\mathrm{T}$ & Af \\
\hline & Vetiveria nigritana (Benth.) Stapf & $\mathrm{Ch}$ & Af \\
\hline POLYGALACEAE (D) & Polygala erioptera DC. & $\mathrm{T}$ & As \\
\hline POLYGONACEAE (D) & Coccoloba uvifera L. & $\mathrm{P}$ & Am \\
\hline PUNICACEAE (D) & Punica granatum L. & $\mathrm{P}$ & As \\
\hline & Ziziphus mucronata Willd. & $\mathrm{P}$ & As \\
\hline RHAMNACEAE (D) & Zizyphus joazeiro C. Mart. & $\mathrm{P}$ & Am \\
\hline & Zizyphus mauritiana Lam. & $\mathrm{P}$ & As \\
\hline RUIRIACFAF (D) & Spermacoce stachydea DC. & $\mathrm{T}$ & Af \\
\hline KUDIACEAL (D) & Spermacoce verticillata $\mathrm{L}$. & $T$ & M \\
\hline RUTACEAE (D) & Zanthoxylum zanthoxyloides (Lam.) Zep. \& Tim & $\mathrm{P}$ & Af \\
\hline & Aphania senegalensis (Juss. ex Poir.) _kRadlk & $\mathrm{P}$ & Af \\
\hline SAPINDACEAE (D) & Dodonae aviscosa (L.) Jacq. & $\mathrm{P}$ & $\mathrm{Pt}$ \\
\hline & Sapindus saponaria L. & $\mathrm{P}$ & $\mathrm{Am}$ \\
\hline SCROPHULARIACEAE (D) & Striga hermonthica (Del.) Benth. & Par & $\mathrm{M}$ \\
\hline SOL ANACEAF (D) & Datura fastuosa $\mathrm{L}$. & $\mathrm{T}$ & $\mathrm{Pt}$ \\
\hline SULAINACEAE (D) & Solanum nigrum L. & $T$ & Cosm \\
\hline TAMARICACEAE (D) & Tamarix senegalensis DC & $\mathrm{P}$ & Af \\
\hline ULMACEAE (D) & Celtis integrifolia Lam. & $T$ & As \\
\hline VEDRENACEAF /DI & Gmelina arborea Roxb. & $T$ & $\mathrm{Pt}$ \\
\hline VERBENACEAE (D) & Lantana camara L. & $\mathrm{P}$ & Pt \\
\hline VITACEAE (D) & Cissus quadrangularis $\mathrm{L}$. & G & As \\
\hline ZYGOPHYLLACEAE (D) & Guaiacum officinale L. & $P$ & Am \\
\hline
\end{tabular}

$\mathrm{D}=$ Dicotylédones $; \mathbf{M}=$ Monocotylédones

Thérophytes (T) ; Hémicryptophytes (Ch) ; Géophytes (G) ; Parasites (Par) ; Chaméphytes (C) ; Nanohanérophytes (P) ; Africaines (Af) ; pantropicales (Pt) ; australiennes (Asu) ; asiatiques (As) ; afro-asiatiques et européennes (Ase) ;

cosmopolites (Cosm) ; afro-malgaches (M) ; afro- malgaches et asiatiques (Mas) ; américaines et asiatiques (AmAs) ; américaines $(\mathrm{Am})$

Tableau 4 : Structure de la flore du Conservatoire botanique Michel Adanson

\begin{tabular}{|l|c|c|c|c|c|c|}
\hline \multirow{2}{*}{} & \multicolumn{2}{|c|}{ Familles } & \multicolumn{2}{c|}{ Genres } & \multicolumn{2}{c|}{ Espèces } \\
\cline { 2 - 7 } & Nombre & $\%$ & Nombre & $\%$ & Nombre & $\%$ \\
\hline Classe des Dicotylédones & 48 & 90,57 & 100 & 86,96 & 120 & 85,71 \\
\hline Classe des Monocotylédones & 5 & 9,43 & 15 & 13,04 & 20 & 14,29 \\
\hline Total & 53 & 100,00 & 115 & 100,00 & 140 & 100,00 \\
\hline
\end{tabular}

Spectre biologique : La figure 3 est une représentation des différentes espèces du Conservatoire botanique regroupées selon le type biologique. Elle indique que $47,86 \%$ de cette flore est constituée de Nanophanerophytes. Ce sont les plus communes dans le Conservatoire. Les thérophytes $(42,86 \%)$ qui se rencontrent dans la plupart des familles (Amaranthaceae,
Acanthaceae, Convolvulaceae, Fabaceae, Poaceae, etc.) viennent en seconde position. Le reste est constitué de 5 hemicriptophytes, de 4 géophytes, de 2 espèces parasites et de 2 chaméphytes.

Spectre chorologique: La figure 4 représente la répartition des espèces du Conservatoire botanique regroupées en fonction de leurs affinités 
biogéographiques. Les espèces africaines $(33,8 \%)$ sont avec les espèces pantropicales $(21,6 \%)$ les plus importantes. Elles forment $55,39 \%$ des espèces recensées. Le reste des espèces est constitué essentiellement par des espèces afro-asiatiques $(16,5 \%)$, des espèces afro-americaines $(10,1 \%)$, des espèces cosmopolites $(6,5 \%)$, des espèces afro-asiatiques et australiennes $(4,3 \%)$, et des espèces afro-malgaches et asiatiques $(3,6 \%)$. Les autres espaces sont peu nombreuses.

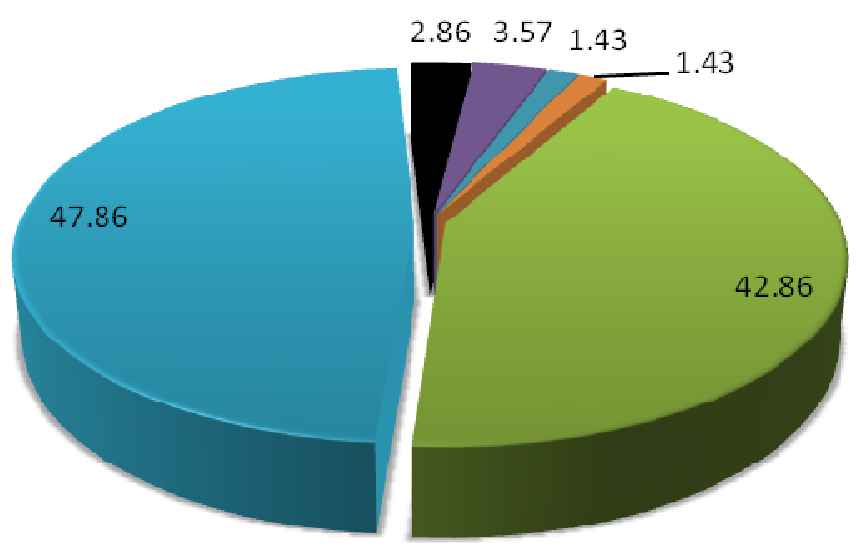

曰 Thérophytes (T)

$\square$ Nanophanérophytes (P)

Q Géophytes (G)

- Hémicryptophytes ( $C_{1}$ )

- Parasites (Par)

n Chaméphytes ( C)

Figure 3 : Représentation du spectre biologique des espèces rencontrées dans le Conservatoire

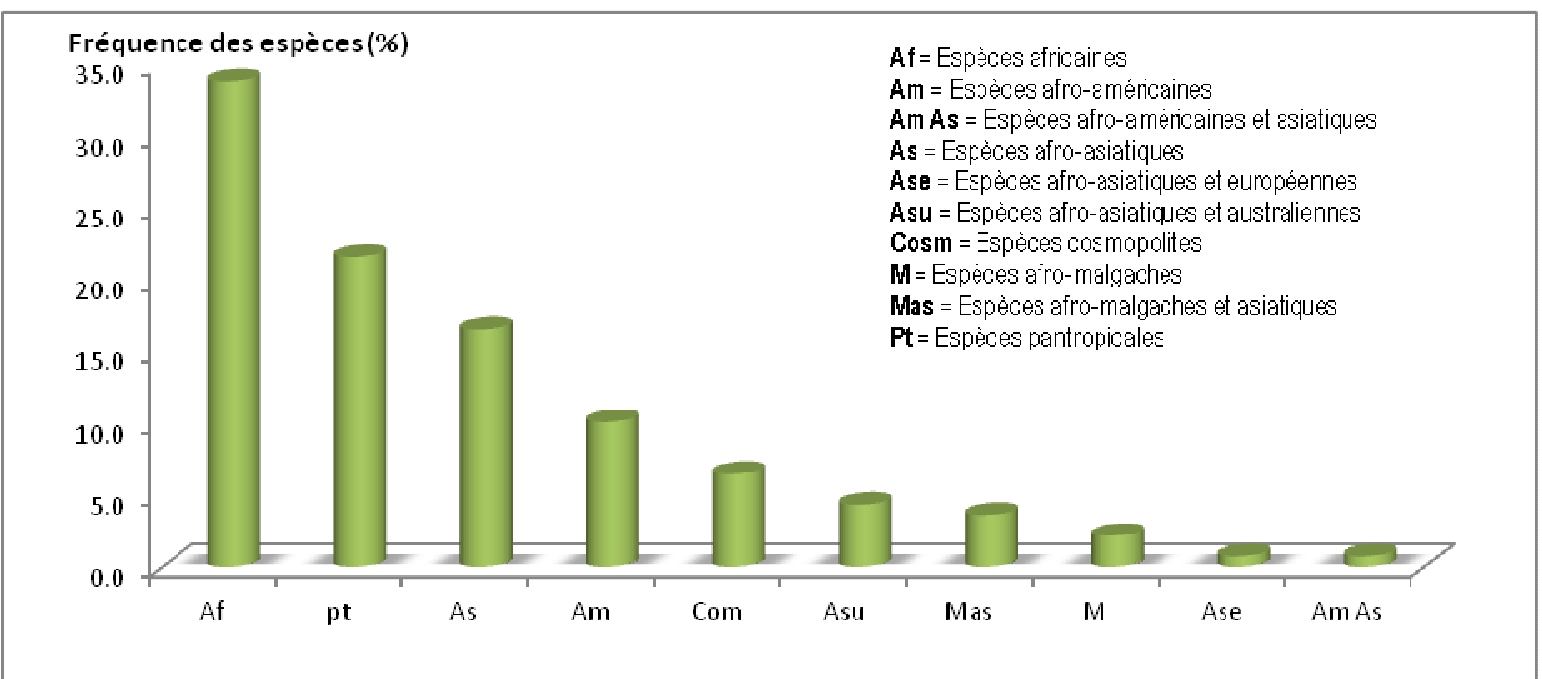

Figure 4 : Représentation du spectre chorologique de la flore du Conservatoire botanique

Fréquences des espèces: Les espèces du Conservatoire botanique Michel Adanson de Mbour sont distribuées dans les différentes parcelles avec une certaine fréquence. Le tableau 6 présente la proportion du nombre d'espèces selon les indices de Caratini. Ces résultats montrent que plus de la moitié des espèces $(59,28 \%)$ recensées sont rares ou accidentelles avec une dominance des espèces ligneuses du fait du caractère $d u$ site qui est un conservatoire. Les espèces accessoires représentent 17,14\% parmi lesquelles : Acacia bivenosa, Acacia tortilis subsp. raddiana, Adansonia digitata, Boerhaavia repens, Celtis integrifolia, Cenchrus biflorus. Les mêmes proportions $(7,86 \%)$ ont été notées au niveau des espèces constantes (Leucaena leucocephala, Achyranthes argentea, Vigna gracilis, Combretum aculeatum, Prosopis juliflora, Pergularia daemia, Casuarina equisetifolia, Merremia pentaphylla), des espèces abondantes (Thephrosia lathyroides, 


\section{Diop et al., J. Appl. Biosci. 2017 La flore du conservatoire botanique Michel Adanson de Mbour (Sénégal) :}

perspective pour un plan d'aménagement et de gestion

Tapinenthus bangwensis, Sesbania pachycarpa, Maytenus senegalensis, Leptadenia hastata, Guaiacum officinale, Grewia bicolor, Commelina benghalensis) et des espèces fréquentes du conservatoire (Azadirachta indica, Cassia occidentalis, Commiphora africana, Hibiscus sabdariffa, Ipomea pes-tigridis, Croton lobatus, Alysicarpus ovalifolius). Les espèces constantes sont présentes dans pratiquement toutes les parcelles. C'est le cas de Leucaena leucocephala qui est rencontré dans toutes les 16 parcelles du conservatoire.
L'Abondance dominance: Les résultats du tableau 7, montrent que $2,26 \%$ des espèces ont un recouvrement moyen de $32 \%$. Ces espèces sont les plus abondantes du conservatoire. C'est le cas d'Achyrantes aspera var. sicula, Justicia ladanoides, Passiflora foetida, Triumfetta pentandra. Environs 16,43\% des espèces ont un recouvrement moyen de $14 \%$ des parcelles du Conservatoire et $27,14 \%$ ont un recouvrement compris entre 1 à $5 \%$ des parcelles. Les espèces ayant un recouvrement insignifiant c'est-à-dire compris entre 0 à $1 \%$ sont majoritaires et représentent $43,57 \%$.

Tableau 5 : Répartition par familles des espèces recensées dans le Conservatoire botanique Michel Adanson.

\begin{tabular}{|l|c|c|}
\hline \multirow{2}{*}{ Familles } & \multicolumn{2}{|c|}{ Flore du conservatoire } \\
\cline { 2 - 3 } & Nombre d'espèces & \% de familles \\
\hline FABACEAE(D) & 27 & 19,29 \\
\hline POACEAE $(\mathrm{M})$ & 13 & 9,29 \\
\hline MALVACEAE (D) & 9 & 6,43 \\
\hline EUPHORBIACEAE (D) & 7 & 5 \\
\hline CONVOLVULACEAE (D) & 6 & 4,29 \\
\hline AMARANTHACEAE (D) & 4 & 2,86 \\
\hline ASTERACEAE (D) & 4 & 2,86 \\
\hline CAPPARIDACEAE (D) & 4 & 2,86 \\
\hline ACANTHACEAE (D) & 3 & 2,14 \\
\hline ASCLEPIADACEAE (D) & 3 & 2,14 \\
\hline MYRTACEAE (D) & 3 & 2,14 \\
\hline NYCTAGINACEAE (D) & 3 & 2,14 \\
\hline RHAMNACEAE (D) & 3 & 2,14 \\
\hline SAPINDACEAE (D) & 3 & 2,14 \\
\hline ANACARDIACEAE (D) & 2 & 1,43 \\
\hline ARECACEAE (M) & 2 & 1,43 \\
\hline BIGNONIACEAE (D) & 2 & 1,43 \\
\hline COMBRETACEAE (D) & 2 & 1,43 \\
\hline COMMELINACEAE (M) & 2 & 1,43 \\
\hline CUCURBITACEAE (D) & 2 & 1,43 \\
\hline CYPERACEAE (M) & 2 & 1,43 \\
\hline RUBIACEAE (D) & 2 & 1,43 \\
\hline SOLANACEAE (D) & 2 & 1,43 \\
\hline VERBENACEAE (D) & 2 & 1,43 \\
\hline ANNONACEAE (D) & 1 & 0,71 \\
\hline BALANITACEAE (D) & 1 & 0,71 \\
\hline BORAGINACEAE (D) & 1 & 0,71 \\
\hline BURSERARACEAE (D) & 1 & 0,71 \\
\hline CACTACEAE (D) & 1 & 0,71 \\
\hline CARICACEAE (D) & 2 & 0,71 \\
\hline CASUARINACEAE (D) & 2 & 0,71 \\
\hline & 2 & \\
\hline
\end{tabular}


Diop et al., J. Appl. Biosci. 2017 La flore du conservatoire botanique Michel Adanson de Mbour (Sénégal) : perspective pour un plan d'aménagement et de gestion

\begin{tabular}{|l|c|c|}
\hline CELASTRACEAE $(\mathrm{D})$ & 1 & 0,71 \\
\hline HIPPOCRATEACEAE (D) & 1 & 0,71 \\
\hline LABIEAE (D) & 1 & 0,71 \\
\hline LILIACEAE(M) & 1 & 0,71 \\
\hline LORANTHACEAE (D) & 1 & 0,71 \\
\hline LYTHRACEAE (D) & 1 & 0,71 \\
\hline MELIACEAE (D) & 1 & 0,71 \\
\hline MENISPERMACEAE (D) & 1 & 0,71 \\
\hline MORACEAE (D) & 1 & 0,71 \\
\hline MORINGACEAE (D) & 1 & 0,71 \\
\hline PASSIFLORACEAE (D) & 1 & 0,71 \\
\hline PEDALIACEAE (D) & 1 & 0,71 \\
\hline POLYGALACEAE (D) & 1 & 0,71 \\
\hline POLYGONACEAE (D) & 1 & 0,71 \\
\hline PUNICACEAE (D) & 1 & 0,71 \\
\hline RUTACEAE (D) & 1 & 0,71 \\
\hline SCROPHULARIACEAE(D) & 1 & 0,71 \\
\hline TAMARICACEAE (D) & 1 & 0,71 \\
\hline ULMACEAE (D) & 1 & 0,71 \\
\hline VITACEAE (D) & 1 & 0,71 \\
\hline ZYGOPHYLLACEAE (D) & 1 & 0,71 \\
\hline TOTAL & 140 & 100 \\
\hline
\end{tabular}

$\mathrm{D}=$ Dicotylédones

$M=$ Monocotylédones

Tableau 6: Proportion du nombre d'espèces selon les indices de Caratini

\begin{tabular}{|c|c|c|}
\hline Indices & Nombre d'espèces & Proportion(\%) \\
\hline V & 11 & 7,86 \\
\hline IV & 11 & 7,86 \\
\hline III & 11 & 7,86 \\
\hline II & 24 & 17,14 \\
\hline I & 83 & 59,29 \\
\hline Total & 140 & 100,00 \\
\hline
\end{tabular}

Tableau 7 : Proportion du nombre d'espèces par rapport à l'abondance/dominance moyenne et le recouvrement moyen

\begin{tabular}{|c|c|c|c|}
\hline ADM & nombre d'espèces & $\%$ & Rec moy \\
\hline 3 & 4 & 2,86 & 32 \\
\hline 2 & 23 & 16,43 & 14 \\
\hline 1 & 38 & 27,14 & 3 \\
\hline $0,1-0,5$ & 75 & 53,57 & $0-1$ \\
\hline Total & 140 & 100,00 & \\
\hline
\end{tabular}

\section{DISCUSSION}

Le nombre d'espèces du conservatoire botanique Michel Adanson est plus important que ceux signalés dans le delta, le lac de Guiers et sa plaine d'inondation par
Trochain (1940) qui a inventorié 79 espèces réparties dans 56 genres et 28 familles et par Thiam (1998) qui fait état de 98 espèces, 74 genres et 38 familles. Cette forte 
biodiversité est due à la nature du site qui est un conservatoire ; donc chaque année de nouvelles espèces sont sélectionnées et introduites dans le site. L'importance relativement élevée de la diversité spécifique des dicotylédones est due au fait que certaines familles y sont très diversifiées, telles que la famille des Fabaceae avec 27 espèces et celle des Malvaceae avec 9 espèces. Ces deux familles forment à elles seules près du tiers des espèces inventoriées. Comparée à la flore vasculaire du Sénégal (Ba et Noba, 2001), la flore du conservatoire botanique Michel Adanson de Mbour présente des proportions comparables entre dicotylédones et monocotylédones. Les dicotylédones représentent $85,71 \%$ contre $70,3 \%$ dans l'ensemble de la flore du Sénégal et les monocotylédones $14,29 \%$ contre $14,7 \%$ pour la flore globale du pays. En ce qui concerne le nombre de famille, la flore du Conservatoire botanique Michel Adanson de Mbour présente approximativement la même structure que la flore adventice dans la zone des Niayes (Sarr et al., 2007) : - certaines familles se trouvent en proportion plus importante dans la flore adventice dans la zone des Niayes que dans la flore du CBMA; c'est le cas des Amaranthaceae, des Poaceae et des Fabaceae;

- d'autres familles sont moins importantes dans la flore adventice dans la zone des Niayes que dans la flore du CBMA, notamment les Nyctaginaceae, les Rhamnaceae et les Acanthaceae;

- les familles des Passifloraceae et des Scrophulariaceae ne sont représentées que par une seule espèce aussi bien dans la flore du CBMA que dans la flore adventice de la zone des Niayes.

Le Conservatoire botanique Michel Adanson de Mbour offre une forte diversité spécifique, dans un espace limité, une faible diversité de milieux écologiques avec une côte sableuse et une savane arbustive. La nature du sol qui est par endroit limoneux à limono-sableux et presque constamment humide grâce un système d'arrosage qui

\section{CONCLUSION}

II ressort de cette étude que la flore du Conservatoire botanique Michel Adanson de Mbour est une flore relativement diversifiée et compte au moins 140 espèces réparties dans 115 genres et 53 familles. Par ordre d'importance, les Fabaceae, Poaceae, Malvaceae, Euphorbiaceae, Convolvulaceae, Amaranthaceae, Capparidaceae sont les mieux représentées puisqu'elles totalisent plus de la moitié des espèces inventoriées. Sur le plan biologique, les nanophanérophytes $(47,86 \%)$ dominent la flore du conservatoire, mais les thérophytes $(42,86 \%)$ sont fortement représentés. Toutefois les est permanant et la variété des espèces plantées fait qu'il constitue un îlot de verdure dans un environnement fortement anthropisé. Comme le montre le spectre biologique, la plupart des espèces sont des nanophanerophytes. Ceci est dû au fait que les espèces pérennes sont plus adaptées à la conservation donc elles sont sélectionnées et introduites dans le site. Elles s'adaptent ainsi à l'instabilité climatique. Les thérophytes ont un cycle de vie parfois très court, de quelques semaines, comme chez les espèces du genre Boerhavia, Eragrostis, et Corchorus, Amaranthus, ou plus long et forment alors une importante quantité de matière vivante ou sèche selon la saison. Leur cycle est généralement synchrone avec la saison des pluies (Noba et al., 2010). Au plan de la répartition biogéographique, les espèces africaines et pantropicales constituent plus de la moitié de la flore (55,39\%). Ces résultats sont comparables à celles observées dans la flore des Niayes (Sarr et al., 2007). La flore $\mathrm{du}$ conservatoire botaniques est plus riche en espèces africaines pantropicales et afro-asiatiques et moins riche en espèces cosmopolites que la flore adventice des Niayes. La présence dans le Conservatoire des espèces d'affinités biogéographiques diverses semble probablement liée à la forte introduction d'espèces venant d'horizons différentes. Leucaena leucocephala présente dans toutes les parcelles est l'espèce la plus constante. Cette espèce plantée dans le site présente une importante capacité de dissémination. La plupart des espèces locales comme exotiques sont circonscrites dans les parcelles où elles sont plantées. Les espèces comme Achyranthes argentea, Justicia kotschyi, Passiflora foetida, Triumfetta pentandra sont plus abondantes dans le conservatoire. Ceci est peut être dû à la capacité de germination des graines et la vitesse de prolifération de ces herbacées pendant l'hivernage. Les espèces les moins abondants sont majoritaires. C'est le cas de la plus part des ligneux qui ne sont présents que dans les espaces où ils sont implantés.

hémicryptophytes $(3,57 \%)$, les géophytes $(2,86 \%)$, les chaméphytes $(1,43 \%)$ et les plantes parasites $(1,43 \%)$ sont présentes dans la flore. Malgré l'homogénéité du milieu, cette flore est caractérisée par une forte présence d'espèces africaines $(33,8 \%)$, pantropicales $(21,6 \%)$, afro-asiatiques (16,5\%). Cependant les espèces afroaméricaines $(10,1 \%)$, les espèces cosmopolites $(6,5 \%)$, les espèces afro-asiatiques et australiennes $(4,3 \%)$, et des espèces afro-malgaches et asiatiques $(3,6 \%)$, les espèces afro-malgaches $(2,2 \%)$, les espèces afroasiatiques et européennes $(0,7 \%)$ et les espèces afro- 
américaines et asiatiques $(0,7 \%)$ y sont représentées. Sur le plan de la fréquence, la moitié des espèces recensées $(59,28 \%)$ sont rares ou accidentelles. Les espèces accessoires représentent $17,14 \%$ tandis que les espèces constantes constituent $7,6 \%$ de la flore. Les estimations sur l'abondance dominance montrent que les espèces les plus abondantes représentent $2,26 \%$. C'est le cas

\section{REMERCIEMENTS}

Les auteurs remercient ENDA-MADESAHEL qui à travers l'assistance financière et la mise à disposition du

\section{REFERENCES BIBLIOGRAPHIQUES}

$\mathrm{Ba} A$. T. et Noba K 2001. Flore et biodiversité végétale au Sénégal, Sécheresse12 (3) : 149-155.

Berhaut J, 1967. Flore du Sénégal. $2^{\text {ème }}$ Ed. Clairafrique, Dakar-Sénégal, 257 pp.

Berhaut J, 1971-1991. Flore illustrée du Sénégal. Ed. Gouvernement du Sénégal, MDR/DEF, 10 tomes.

Gillet $F, 2000$. La phytosociologie synoviales intégrée: Gide méthodologique, Université de NeuchâtelInstitut de Botanique. Docu. Labo. Ecol. Vég. 68pp.

www.madesahel.enda.sn/index.html. 25/08/2003 à $12 \mathrm{~h}$.

Hutchinson J. et Dazeil Jm 1954. Flora of West tropical Africa Vol 1 Part 1. \& $22^{\text {nd }}$ ed. Revised by R. W.J. Keay. \& al., Thewhitefriars. Press Ldt, London, Tonbridge, England, 828pp.

Hutchinson J. et Dazeil Jm 1963. Flora of West tropical Africa Vol 2, $2^{\text {nd }}$ ed. Edited by F. N. HEPPER. \& al., Thewhitefriars. Press Ldt, London, Tonbridge, England, 544pp.

Hutchinson J. et Dazeil Jm 1968. Flora of West tropical Africa Vol 3 Part $1 \& 2,2^{\text {nd }}$ ed. Revision edited By F. N. Hepper. hewhitefriars. Press Ldt, London, Tonbridge, England, 574pp.

Kahlem G, 1981. La végétation de la forêt de Bandia: évolution des populations végétales et de la production de graine pendant l'année 1978, 1979, 1980, bulletin de l'IFAN.

Lebrun J, 1966. Les formes biologiques dans les végétations tropicales. Bull. Soc. Bot. de France : 164-175.

Mugnier J. 2008. Nouvelle flore du Sénégal et des régions voisines.
d'Achyrantes aspera var. sicula, Justicia ladanoides, Passiflora foetida, Triumfetta pentandra. Les espèces ayant un recouvrement insignifiant donc les moins abondants sont majoritaires et représentent $43,57 \%$. C'est le cas de la plus part des ligneux plantés dans le site.

conservatoire botanique Michel Adanson de Mbour, a facilité la réalisation de ce travail.

Noba K, 2002. La flore adventice dans le sud du bassin arachidier (Sénégal) : structure, dynamique et impact sur la production du mil et de l'arachide. Thèse de doctorat d'État, Université Cheikh Anta Diop, Dakar-Sénégal, 137pp.

Noba K, Mbaye Ms, Coundoul M, Kane A, Hane Pd, Ba N, Mbaye N, Guisse A, Faye Mn, Ba At 2010. La flore du Parc national des oiseaux de Djoudj une zone humide du Sénégal. Sécheresse 2010: vol. $21, n^{\circ} 1$.

Raunkiaer C, 1934. The life forms of plants and statistical Plants Geography Clarendron, Press, Oxford. $623 p p$.

Sambou B, 2004. Évaluation de l'État, de la dynamique et des tendances évolutives de la flore et de la végétation ligneuses dans les domaines soudanien et sub-guinéen du Sénégal. Thèse de Doctorat d'État, Université Cheikh Anta Diop, Dakar-Sénégal, 210pp.

Sarr RS, Mbaye MS, Ba AT 2007. Flore adventice des cultures d'oignon dans la zone périurbaine de Dakar (Niaye) Sénégal, Webbia 62 (2) : 205216.

Thiam A, 1998. Flore et végétation aquatiques et des zones inondables du delta du fleuve Sénégal et le lac de Guiers. AAU Reports : 39 : 245-57.

Tine M, 2009. Analyse des impacts socio-economiques et spatiaux du PAPA-SUD dans la Petite Côte; cas de Mbour et Joal, Master I de l'Université Gaston Berger de Saint Louis (Sénégal), 87pp.

Trochain J, 1940. Contribution à l'étude de la végétation du Sénégal. Mémoires de l'IFAN, 2 : 433pp. 